\title{
Using science to improve the health and well-being of older women
}

\author{
Nature Aging speaks with Paula Rochon, geriatrician and advocate for women's healthy aging, about the specific \\ health needs of older women and gendered ageism in health and social structures. Rochon reflects on her \\ motivations and career, and shares her plans as the founding director of the Women's Age Lab to help to improve \\ health and social care to positively impact the health and well-being of older women.
}

\begin{abstract}
As a researcher who has devoted her career to improving health and healthcare for older adults, what is your definition of aging?

Aging, simply put, is the process of getting older. It is associated with biological and sociocultural changes. Traditionally, 'older adult' is defined as over the age of 65 based on retirement age, but everyone's interpretation is different and highly subjective. Is it over 50 ? Is it over 65 ? Or is it when you are approaching 100 ? For many it is relative. People often consider 'old' simply as being older than they are. People are hesitant to embrace the word 'old'.

From an aging perspective, we are all aging - it's not just happening to other people, it is happening to all of us. This is something people often don't seem to appreciate. Aging will impact all of us and if you are lucky, you will be able to experience the process of aging. It is a privilege rather than a burden.
\end{abstract}

\section{"Aging will impact all of us and if you are lucky, you will be able to experience the process of aging."}

Much of your research has focused on the unique needs of older women: what shaped this interest and career for you, did you have any role models?

I am a geriatrician and have always been interested in older people. My grandfather lived to almost 103 and my grandmother well into her nineties, both were very healthy and their lives inspired me to look more closely at what it is we can do to promote healthy aging. One of my first studies while doing my MPH (Master of Public Health) degree documented how older people were not adequately included in drug trials of arthritis medications. Arthritis is a condition that disproportionately impacts older people, particularly older women. Addressing this underrepresentation and underreporting of information, particularly

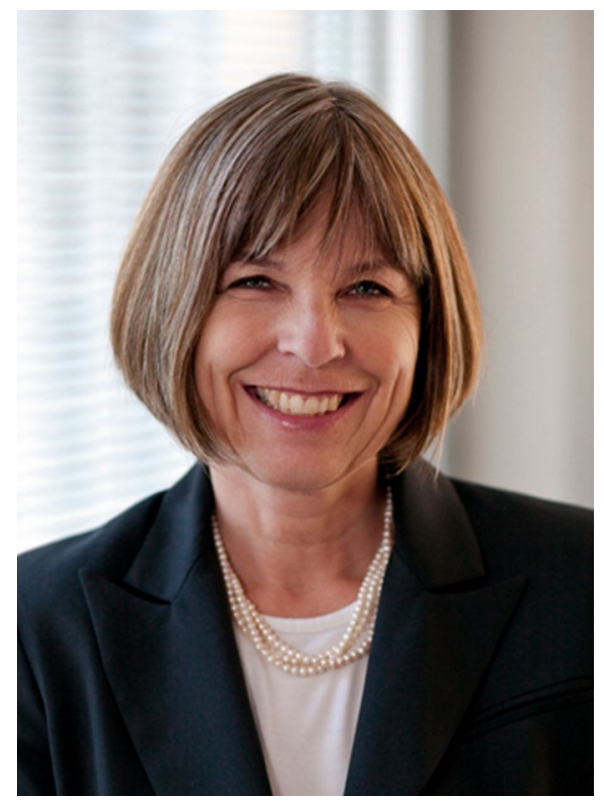

Credit: Paula Rochon

about older women, in research has continued to be a theme in my work.

Can you comment on the representation of older people and the visibility of women as participants in clinical research on aging and age-related diseases? Has there been change, and where do we still fall short?

If older women are going to be using the therapies or the intervention, then they need to be included in the related research. It is difficult to imagine that women weren't required to be included in clinical trials until the 1990s by the National Institutes of Health (NIH), and up until 2019, older adults were not required to be included in NIH-funded studies. Lack of available data disaggregated by sex and age caused disparities in understanding the needs of older women and the ability to create tailored interventions for them. Further, this masks opportunities to explore important sex and gender differences. While we wait for research and reporting standards to catch up, post-marketing surveillance using large administrative datasets is a great way to explore the issues in a real-world population. In these studies, such as those related to prescribing cascades that we are conducting in Canada, we are able to study the entire population - including data on older women. There is a vacuum in clinical research on the intersection between age and sex, and a lack of consideration of gender-related factors. This gap is a missed opportunity to better understand the needs of older women and men. During the COVID-19 pandemic, only three of the ten countries with the most cases reported data disaggregated by sex and age, which means that valuable insights and information are lost (Rochon, P. A. et al. Lancet, 397, 21; 2021, and Rochon, P. A. et al. J. Am. Geriatr. Soc. 68, 1671-1673; 2020). This sex and age data gap rendered women aged 65 years or older largely invisible and their unique care needs unmet. Our work is anchored in raising awareness around the value and importance of using data disaggregated by sex and age to produce evidence-informed policy making, and improve health and social care for older women.

\section{"There is a vacuum in clinical research on the intersection between age and sex, and a lack of consideration of gender-related factors." \\ "Our work is anchored in raising awareness around the value and importance of using data disaggregated by sex and age to produce evidence- informed policy making:"}

Aging is far from being a uniform process; how do women and men experience aging differently? 
It is critical that we look at older women as distinct from older men, rather than considering them as the homogenous category of 'older people'. Older women face multiple layers of discrimination - not only do they face discrimination by age, but also by sex and as a result of gender-related differences, which has farreaching consequences for their health and well-being.

Such gendered ageism is deeply embedded in contemporary culture and social and economic policies, often rendering older women largely invisible. Gendered ageism is institutionalized in healthcare practices and socioeconomic policies. A lifetime of discrimination towards women leads to a poverty gap when they become older. In countries of the OECD (Organisation for Economic Co-operation and Development), older women receive $26 \%$-smaller pensions (https://go.nature.com/3fDvIQR) than men, impacting their future health and independence.

Some medical conditions present more commonly in women (such as thyroid disease) and others can in some cases present with different symptoms in women (such as myocardial infarction). Differences also occur in the related drug therapies used to manage these conditions. For example, differences in prescribing behavior have been observed, where women are more likely to be treated by physicians for mental health conditions with psychoactive medications and men are more likely to receive secondary prevention for cardiac conditions. On average, older women are more likely than men to suffer from multiple medical problems, chronic conditions, take more medications and be prescribed medications that are inappropriate, resulting in adverse events; thus, we need to consider how these differences can inform better prescribing decisions (Rochon, P. A. et al. Lancet Healthy Longev. 2, 290-300; 2021), particularly for women.

Women make up the majority of longterm care residents and are at higher risk of experiencing loneliness (often outliving their spouses). From the Canadian Longitudinal Study on Aging (https://go.nature. $\mathrm{com} / 3 \mathrm{tDHkeO}$ ), about $40 \%$ of women aged 65 years and older who live alone report being lonely, translating to approximately 380,000 older women who live alone and are lonely in Canada. The better question is 'how are the experiences of women and men not distinct?'. As a society, we need to ensure that the needs of older women are clear and ensure that they do not become 'invisible' as they age.

\section{"As a society, we need to ensure that the needs of older women are clear and ensure that they do not become 'invisible' as they age."}

\section{Women are more likely to assume caregiving roles. Can this exacerbate women's health disparities?}

Older women on average spend more time on caregiving activities (as per Statistics Canada (https://go.nature. com/3s2onA8)), and report more stress and impact on their health and life as a result of those responsibilities. Caregiving is an intergenerational issue as the lifelong accumulation of disadvantage for women related to caregiving activities from being less likely to enter the paid workforce, maternity leave and fewer career advancement opportunities - results in reduced lifetime earnings and subsequently smaller pensions. This leads to a situation where older women are more likely to be living in poverty than older men, and thus less likely to afford costly dental care, vision care, hearing aids, physiotherapy and other services not covered entirely by the government or private health-insurance plans. As a result, older women are left at serious risk of deterioration in their health. Older women are also generally more likely to live alone and tend to outlive their spouses, leaving them without a caregiver themselves.

\section{Where do we meet the specific needs of women to support healthy aging and where do we fall short?}

There is agreement that older women have unique health needs that need to be considered, and we now need an action plan to put this idea into practice and identify solutions to close the gap that older women currently face in our system. We need to think about empowering women and provide them with the necessary resources to promote health and well-being with aging. Healthcare reform for older adults tends to focus on long-term care, but we need to remember that more than $90 \%$ of older people are healthy and living in the community. Compared to previous generations, older adults are maintaining higher levels of independence and remaining healthier, longer. Given that this vast majority of older adults live in the community, it is important to tailor support to meet their specific needs that will enable them to age in the place they choose.

\section{"There is agreement that older women have unique health needs that need to be considered, and we now need an action plan to put this idea into practice and identify solutions to close the gap that older women currently face in our system."}

\section{You are the founding director of the Women's Age Lab. What are the mission and plans of the Women's Age Lab? How do you go about integrating gender, eth- nicity, income and other intersectionality into your work at the Women's Age Lab?} My decades of research that studies older adults, particularly women, led to my founding of the Women's Age Lab (https:// go.nature.com/3fzVAgC), to our knowledge, the first and only center of its kind in the world, whose mission is to improve the lives of older women by using science to transform care and practice, and drive health-system and social change.

The work of the Women's Age Lab will focus on four key priority areas: addressing gendered ageism (that is changing the way we think, feel and act toward older women); reimagining aging in place and congregate care; optimizing therapies; and promoting social connectedness. In all of this we have the cross-cutting goal of collecting and using sex- and agedisaggregated data. These priority areas are rooted in international initiatives such as the WHO Decade of Healthy Aging (https:// go.nature.com/3nGg9MJ), Global Campaign to Combat Ageism (https://go.nature. com/3qFpI0c) and Medication without Harm (https://go.nature.com/3Ac3kyN).

Our strategic objectives include identifying and studying issues in health and social care; closing the 'know-do' gap, by testing practical and scalable solutions; and inspiring new ways of thinking, and scienceinformed advocacy.

Women's Age Lab is committed to building an inclusive, just and equitable community that values, supports and honors the wisdom, lived experience and contributions of all people. Our work is intended to benefit people of all genders, ethnicities and socioeconomic backgrounds. We use a 'Sex and Gender-Based Analysis Plus' analytic approach in our research. We are aiming to collaborate with organizations and networks in academia and healthcare to inform our approach to address issues distinct to older 
women from different backgrounds. Part of our work will be focused on advancing our research in this domain.

\section{How do you foster an action-oriented} program?

We will foster an action-oriented program through initiatives at a local (hospital), community and global level with defined deliverables to measure progress. Locally, the Women's Age Lab is working on collecting sex- and age-disaggregated data to determine trends and patterns related to older women visiting the hospital, explore opportunities to improve health and social care, and support and create best practices for all hospitals to use sexand age-disaggregated data to inform care planning. We are hoping to create best practices for other hospitals and healthcare institutions to collect and use sex- and agedisaggregated data for their own patient population. At a community level, we are building partnerships and collaborating with academic and healthcare organizations to improve care and service delivery for older women. On a global level we are supporting global movements on healthy aging, actively publishing articles in high-impact journals and platforms, and exploring opportunities to address gendered ageism.

You highlighted in one of your articles that policies that require the inclusion of individuals of all ages in research may be missing an important opportunity to promote a focus on the intersection between sex, gender and age. This is one example of room for further improvements. If you could make one change in policies today what would you chose?

I would require researchers to report sex- and age-disaggregated data. Such an approach to data collection should be included as part of international data standards and should be considered a requirement for national funding applications, publication on high-impact platforms and beyond. These data will strengthen evidence informed decisionmaking in the clinical and policy realm and promote gender equity in healthy aging.

\section{Interviewed by Anna Kriebs}

Published online: 3 February 2022 https://doi.org/10.1038/s43587-022-00179-y 